\title{
Sensitivity Analysis of Coexistence in Ecological Communities: Theory and Application Supporting Information
}

\author{
György Barabás ${ }^{1}$, Liz Pásztor $^{2}$, Géza Meszéna $^{3}$, \& Annette Ostling ${ }^{4}$ \\ 1: Department of Ecology \& Evolution, University of Chicago \\ 1101 E 57th St, Chicago, IL 60637, USA \\ 2: Department of Genetics, Eötvös Loránd University \\ Pázmány Péter sétány 1C, H-1117, Budapest, Hungary \\ 3: Department of Biological Physics, Eötvös Loránd University \\ Pázmány Péter sétány 1A, H-1117, Budapest, Hungary \\ 4: Department of Ecology and Evolutionary Biology, University of Michigan \\ 830 North University, Ann Arbor, MI 48109-1048
}

\section{Contents}

Sensitivity analysis of forb-grass competition $\quad 2$

Model description . . . . . . . . . . . . . . . . . . . 2

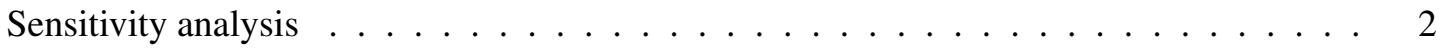

$\begin{array}{lr}\text { The tolerance-fecundity tradeoff model } & 8\end{array}$

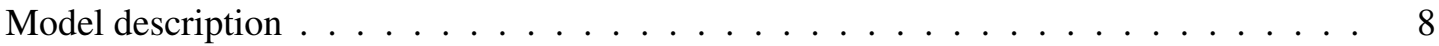

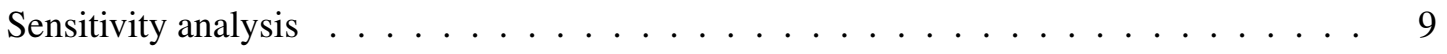

The Gross model $\quad 16$

Model description . . . . . . . . . . . . . . . . . . . 16

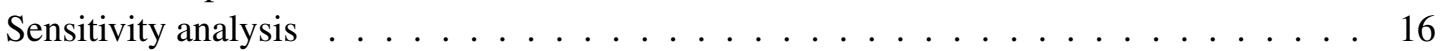

The bound on the number of coexisting species $\ldots \ldots \ldots \ldots \ldots$

Appendix: the volume spanned by a set of vectors $\quad 21$ 


\section{Sensitivity analysis of forb-grass competition}

\section{Model description}

The model of Levine \& Rees (2004) is a discrete-time annual plant model. The equations read

$$
N_{i}(t+1)=\left(\left(1-g_{i}(t)\right)\left(1-d_{i}\right)+\frac{\lambda_{i}(t) g_{i}(t)}{1+\alpha_{i}\left(g_{1}(t) N_{1}(t) / \alpha+g_{2}(t) N_{2}(t)\right)}\right) N_{i}(t),
$$

where the species index $i$ may be 1 (forb) or 2 (grass), and $N_{i}(t)$ is the number of seeds of species $i$ in the seed bank in year $t$ prior to germination. The vector $\alpha_{i}=(\alpha, 1)$ is shorthand for $\alpha$ when $i=1$ and 1 when $i=2$. The description and numerical value of each parameter are given in Table S1. In this model we assume that the fecundities and germination rates fluctuate periodically between "good" and "bad" years.

\begin{tabular}{llr}
\hline $\mathbb{E}$ & Description & Value \\
\hline$d_{1}$ & Forb death rate in the seed bank & 0.1 \\
$d_{2}$ & Grass death rate in the seed bank & 0.7 \\
$g_{1}^{-}$ & Fraction of forb seeds germinating in a bad year & 0.1 \\
$g_{1}^{+}$ & Fraction of forb seeds germinating in a good year & 0.7 \\
$g_{2}^{-}$ & Fraction of grass seeds germinating in a bad year & 0.9 \\
$g_{2}^{+}$ & Fraction of grass seeds germinating in a good year & 0.9 \\
$\lambda_{1}^{-}$ & Number of forb seeds/individual in a bad year & 5 \\
$\lambda_{1}^{+}$ & Number of forb seeds/individual in a good year & 30 \\
$\lambda_{2}^{-}$ & Number of grass seeds/individual in a bad year & 5 \\
$\lambda_{2}^{+}$ & Number of grass seeds/individual in a good year & 30 \\
$\alpha$ & Reciprocal interspecific competition coefficient & 2 \\
\hline
\end{tabular}

Table S1: Numerical value and description of each parameter in the Levine-Rees model. The environment is assumed to oscillate between good (+) and bad ( - ) years, as shown by the variation in the fecundities and germination probabilities. The symbol $\mathbb{E}$ stands for any one model parameter.

The expression in parentheses in Eq. (S1) is the annual geometric growth rate of species $i$; its natural $\log$ is the growth rate $r_{i}(t)$ :

$$
r_{i}(t)=\log \left(\left(1-g_{i}(t)\right)\left(1-d_{i}\right)+\frac{\lambda_{i}(t) g_{i}(t)}{1+\alpha_{i}\left(g_{1}(t) N_{1}(t) / \alpha+g_{2}(t) N_{2}(t)\right)}\right) .
$$

\section{Sensitivity analysis}

Here we go through all the steps of calculating the sensitivity of the stationary state to each model parameter. The steps required for the analysis are outlined in the Box. 


\section{Box: The steps of community-wide sensitivity analysis}

- Step 0: Determine whether the model is in discrete or continuous time, whether the populations are structured, and what type of equilibrium (fixed point, limit cycle, ...) is under consideration.

- Step 1: Designate the regulating factors.

- Step 2: Based on Step 0, look up the necessary formulas for the impact and sensitivity vectors, and calculate them for each species.

- Step 3: Calculate the volumes $\mathcal{V}_{I}$ and $\mathcal{V}_{\mathcal{S}}$. A small product $\mathcal{V}_{\mathcal{I}} \mathcal{V}_{\mathcal{S}}$ signals an oversensitive system. For more precise quantitative estimates, move on to Step 4.

- Step 4: Calculate $a_{i j}$ using the formula appropriate for the particular dynamical scenario under consideration.

- Step 5: Pick an arbitrary model parameter $\mathbb{E}$ of interest and obtain the vector $z_{j}$ from the appropriate formula.

- Step 6: Calculate the sensitivities $\sigma_{i}$ from the general equation

$$
\sigma_{i}=-\sum_{j=1}^{S} a_{i j}^{-1} z_{j},
$$

where $a_{i j}^{-1}$ refers to the $(i, j)$ th entry of the inverse matrix, as opposed to the inverse of its $(i, j)$ th entry.

Step 0. As mentioned before, we assume the environment alternates regularly between good and bad years. This will generate a stationary two-cycle. If the model has a two-cycle, the twicecompounded model has a fixed point. Solving for the fixed point yields two possible solutions, corresponding to the two distinct points within the cycle. Using Eq. (S2), the total population growth over two time steps is zero:

$$
\begin{aligned}
& \log \left(\left(1-g_{i}(0)\right)\left(1-d_{i}\right)+\frac{\lambda_{i}(0) g_{i}(0)}{1+\alpha_{i}\left(g_{1}(0) N_{1}(0) / \alpha+g_{2}(0) N_{2}(0)\right)}\right) \\
& +\log \left(\left(1-g_{i}(1)\right)\left(1-d_{i}\right)+\frac{\lambda_{i}(1) g_{i}(1)}{1+\alpha_{i}\left(g_{1}(1) N_{1}(1) / \alpha+g_{2}(1) N_{2}(1)\right)}\right)=0 .
\end{aligned}
$$


Expressing $N_{i}(1)$ through $N_{i}(0)$ by setting $t=0$ in Eq. (S1), and substituting the result into Eq. (S3), we get

$$
\begin{aligned}
& \log \left(\left(1-g_{i}(0)\right)\left(1-d_{i}\right)+\frac{\lambda_{i}(0) g_{i}(0)}{1+\alpha_{i}\left(g_{1}(0) N_{1}(0) / \alpha+g_{2}(0) N_{2}(0)\right)}\right) \\
& +\log \left(\left(1-g_{i}(1)\right)\left(1-d_{i}\right)+\lambda_{i}(1) g_{i}(1)\right. \\
& \times\left[1+\frac{\alpha_{i} g_{1}(1)}{\alpha}\left(\left(1-g_{1}(0)\right)\left(1-d_{1}\right)+\frac{\lambda_{1}(0) g_{1}(0)}{1+g_{1}(0) N_{1}(0)+\alpha g_{2}(0) N_{2}(0)}\right)\right. \\
& \left.\left.+\alpha_{i} g_{2}(1)\left(\left(1-g_{2}(0)\right)\left(1-d_{2}\right)+\frac{\lambda_{2}(0) g_{2}(0)}{1+g_{1}(0) N_{1}(0) / \alpha+g_{2}(0) N_{2}(0)}\right)\right]^{-1}\right)=0 .
\end{aligned}
$$

This constitutes one equation for $N_{1}(0)$ and $N_{2}(0)$ each, giving the fixed point of the twicecompounded model (i.e., the limit cycle of the original one). With the parameters given in Table S1, the solution can be obtained numerically via any reputable algorithm for solving systems of nonlinear algebraic equations. The two pairs of solutions are

$$
\begin{array}{ll}
\left(N_{1}^{-}=23.636 ;\right. & \left.N_{2}^{-}=10.044\right), \\
\left(N_{1}^{+}=19.697 ;\right. & \left.N_{2}^{+}=4.329\right),
\end{array}
$$

as is easily verified by substituting these values back into Eq. (S4) along with the parameter values in Table S1 (naturally, there will be some rounding error involved). The $N_{i}^{-}$and $N_{i}^{+}$are the population densities along the two-cycle. Though the literal calculation we performed was for obtaining the densities in year $t=0$, we got two different results because it is completely arbitrary whether we designate $t=0$ to be a bad or a good year. This means that we can interpret $N_{i}^{-}$as the densities in a bad year, and $N_{i}^{+}$as the densities in a good year.

As this is a discrete-time model with unstructured populations, and since the attractor under consideration is a limit cycle, the formulas needed for the analysis are

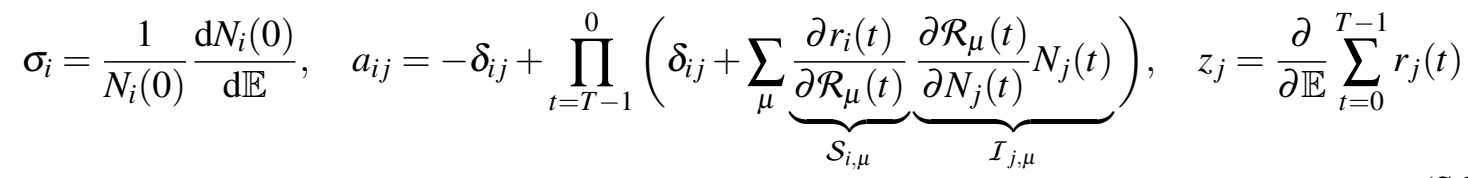

(Barabás \& Ostling 2013), where $\delta_{i j}$ is the identity matrix, equal to 1 if $i=j$ and to 0 otherwise. These quantities are connected through the general sensitivity formula

$$
\sigma_{i}=-\sum_{j=1}^{S} a_{i j}^{-1} z_{j}
$$

where $a_{i j}^{-1}$ is the $(i, j)$ th entry of the inverse matrix, not the inverse of its $(i, j)$ th entry. 
Step 1 . We choose the regulating factors. It is actually possible to designate a single timedependent regulating factor for this model:

$$
\mathcal{R}(t)=\frac{g_{1}(t) N_{1}(t)}{\alpha}+g_{2}(t) N_{2}(t) .
$$

The growth rates in Eq. (S2) read, as a function of this regulating factor, as

$$
r_{i}(t)=\log \left(\left(1-g_{i}(t)\right)\left(1-d_{i}\right)+\frac{\lambda_{i}(t) g_{i}(t)}{1+\alpha_{i} \mathcal{R}(t)}\right) .
$$

Keeping $\mathcal{R}(t)$ constant, we see that these growth rates would become density-independent, therefore our choice for the regulating factors is valid.

Step 2. We calculate the impact and sensitivity vectors. From their definitions in Eq. (S6):

$$
\begin{gathered}
\mathcal{I}_{j}(t)=\frac{\partial \mathcal{R}(t)}{\partial N_{j}(t)}=\frac{g_{j}(t)}{\alpha_{j}} \\
\mathcal{S}_{i}(t)=\frac{\partial r_{i}(t)}{\partial \mathcal{R}(t)}=\left(\left(1-g_{i}(t)\right)\left(1-d_{i}\right)+\frac{\lambda_{i}(t) g_{i}(t)}{1+\alpha_{i} \mathcal{R}(t)}\right)^{-1} \frac{\alpha_{i} \lambda_{i}(t) g_{i}(t)}{\left(1+\alpha_{i} \mathcal{R}(t)\right)^{2}} .
\end{gathered}
$$

Step 3. The volumes spanned by the impact and sensitivity vectors may now be calculated using Eq. (S69) (see the Appendix). We do not perform this step here (we will do so for our other two model examples); instead, we go straight to the detailed sensitivity analysis.

Step 4. The matrix $a_{i j}$ in Eq. (S6) is written for our model as

$$
a_{i j}=-\delta_{i j}+\prod_{t=1}^{0}\left(\delta_{i j}+\frac{\partial r_{i}(t)}{\partial \mathcal{R}(t)} \frac{\partial \mathcal{R}(t)}{\partial N_{j}(t)} N_{j}(t)\right),
$$

or, writing out the matrix product, as

$$
a_{i j}=-\delta_{i j}+\sum_{k=1}^{2}\left(\delta_{i k}+\frac{\partial r_{i}(1)}{\partial \mathcal{R}(1)} \frac{\partial \mathcal{R}(1)}{\partial N_{k}(1)} N_{k}(1)\right)\left(\delta_{k j}+\frac{\partial r_{k}(0)}{\partial \mathcal{R}(0)} \frac{\partial \mathcal{R}(0)}{\partial N_{j}(0)} N_{j}(0)\right) .
$$

We substitute into this expression the equations for the impact and sensitivity vectors, the parameter values in Table S1, and the stationary densities in Eq. (S5). We get two different results depending on whether the initial moment $t=0$ of the cycle is a bad or a good year. If it is a bad year, we get

$$
a_{i j}^{-}=-\left(\begin{array}{ll}
0.449 & 0.079 \\
0.641 & 0.818
\end{array}\right)
$$

Alternatively, if $t=0$ is a good year, we get

$$
a_{i j}^{+}=-\left(\begin{array}{cc}
0.461 & 0.283 \\
0.196 & 0.805
\end{array}\right)
$$




\begin{tabular}{lrrrr}
\hline $\mathbb{E}$ & $z_{1}^{-}$ & $z_{2}^{-}$ & $z_{1}^{+}$ & $z_{2}^{+}$ \\
\hline$d_{1}$ & -0.819 & 0.623 & -1.329 & 0.024 \\
$d_{2}$ & 0.062 & -0.199 & 0.001 & -0.243 \\
$g_{1}^{-}$ & -0.176 & -0.181 & -0.831 & -0.980 \\
$g_{1}^{+}$ & -0.319 & -0.825 & -0.298 & -0.175 \\
$g_{2}^{-}$ & 0.119 & -0.318 & -0.026 & -0.495 \\
$g_{2}^{+}$ & -0.297 & 0.605 & -0.311 & 0.181 \\
$\lambda_{1}^{-}$ & 0.003 & -0.003 & 0.006 & 0 \\
$\lambda_{1}^{+}$ & 0.026 & 0 & 0.026 & -0.003 \\
$\lambda_{2}^{-}$ & -0.050 & 0.125 & 0 & 0.186 \\
$\lambda_{2}^{+}$ & 0 & 0.033 & -0.001 & 0.008 \\
$\alpha$ & -0.153 & 0.328 & -0.152 & -0.134 \\
\hline
\end{tabular}

Table S2: The two components of the vector $z_{j}$ for each parameter. The superscript of $z_{j}$ is “_" if the initial moment of the cycle is a bad year, and " + " if it is a good year.

\begin{tabular}{lrrrr}
\hline $\mathbb{E}$ & $\mathrm{d} N_{1}^{-} / \mathrm{d} \mathbb{E}$ & $\mathrm{d} N_{2}^{-} / \mathrm{d} \mathbb{E}$ & $\mathrm{d} N_{1}^{+} / \mathrm{d} \mathbb{E}$ & $\mathrm{d} N_{2}^{+} / \mathrm{d} \mathbb{E}$ \\
\hline$d_{1}$ & -53.81 & 25.59 & -67.16 & 3.73 \\
$d_{2}$ & 4.98 & -4.11 & 4.33 & -1.54 \\
$g_{1}^{-}$ & -9.68 & 1.01 & -24.45 & -3.96 \\
$g_{1}^{+}$ & -14.61 & -5.26 & -11.89 & -0.31 \\
$g_{2}^{-}$ & 9.15 & -6.96 & 7.41 & -3.06 \\
$g_{2}^{+}$ & -21.78 & 14.69 & -18.77 & 1.98 \\
$\lambda_{1}^{-}$ & 0.20 & -0.11 & 0.28 & -0.02 \\
$\lambda_{1}^{+}$ & 1.58 & -0.53 & 1.34 & -0.09 \\
$\lambda_{2}^{-}$ & -3.79 & 2.80 & -3.28 & 1.18 \\
$\lambda_{2}^{+}$ & -0.20 & 0.47 & -0.18 & 0.05 \\
$\alpha$ & -11.32 & 7.81 & -9.99 & 1.26 \\
\hline
\end{tabular}

Table S3: Sensitivity of forb and grass densities at the initial moment of the cycle to parameter perturbations. The superscript of $N_{i}$ is "-" if the initial moment of the cycle is a bad year, and "+" if it is a good year.

Step 5. To obtain the $z_{j}$ from Eq. (S6), we need the sum of the growth rates for the two points of the cycle. This is given by the left hand side of Eq. (S4). We then take the derivative of this expression with respect to each parameter in turn and substitute numerical values from Table S1 and Eq. (S5) into the results to get the $z_{j}$. Again, two different sets of results emerge depending on whether we designate $t=0$ to be a bad or a good year. Table S2 contains the results.

Step 6. The cycle's sensitivity to each parameter can now be obtained via Eq. (S7). That is, if $t=0$ is a bad year, we multiply the vector $z_{j}^{-}$(given in Table S2) by the inverse of the matrix $a_{i j}^{-}$ 
times minus one. Since the sensitivity $\sigma_{i}$ is given by the derivative of the densities divided by the densities (Eq. S6), we multiply the result by $N_{i}^{-}$(given in Eq. S5) to obtain $\mathrm{d} N_{i}(0) / \mathrm{d} \mathbb{E}$. If $t=0$ is a good year, then we multiply $z_{j}^{+}$by the inverse of $a_{i j}^{+}$and then multiply by minus one times $N_{i}^{+}$. See Table S3 for all sensitivity values.

As a final remark, we emphasize again that coexistence in this model is maintained purely by environmental fluctuations. Using the framework and terminology of Chesson, coexistence is maintained by pure storage effect (Chesson \& Warner 1981, Chesson 1994, 2000). Relative nonlinearity is not operating because $\mathcal{R}(t)$ is a linear function of the population densities (Eq. S8), and fluctuation-independent mechanisms are also not operating because in the absence of fluctuations there is just a single regulating factor and therefore no coexistence. ${ }^{*}$ Therefore the only stabilizing mechanism is the temporal storage effect. We highlight that our sensitivity approach to understanding coexistence differs from Chesson's in that Chesson uses the invader's long-term growth rate to see whether coexistence is possible via mutual invasibility, while the sensitivity approach quantifies coexistence via the range of parameters that will allow for it. See Barabás et al. (2012, Appendix 3) and Barabás \& Ostling (2013, Section 4) for the precise mathematical relationship between the two frameworks.

\footnotetext{
${ }^{*}$ A single regulating factor means the impact and sensitivity vectors are confined to a one-dimensional space. The volume spanned by the vectors of more than one species is therefore necessarily zero, leading to loss of robustness and the breakdown of coexistence.
} 


\section{The tolerance-fecundity tradeoff model}

\section{Model description}

Consider a set of sites, each of which may be occupied by a single sessile individual. The sites vary in the local stress level $s_{a}$, where $a$ runs from 1 to $M: s_{1}$ is the stress level of the least stressful site and $s_{M}$ is that of the most stressful, with various gradations in between (by choosing a sufficiently large $M$, the classification of stress levels can be made arbitrarily fine-grained). Individuals produce seeds that disperse into all sites with a uniform probability distribution. Their fecundities are high enough that no site remains empty after reproduction. Once a seed reaches a vacant site, it has to survive the local stress conditions. Among the seeds that do survive, a lottery draw decides who wins the site. Regardless of stress level, once an individual wins a site, it cannot be displaced except by natural death happening at a species-specific mortality rate.

Assuming that the community consists of $S$ species, this model may be written as

$$
\frac{\mathrm{d} N_{i, a}}{\mathrm{~d} t}=f_{i} N_{i} Q_{i}\left(s_{a}\right)-m_{i} N_{i, a} \quad(i=1 \ldots S),
$$

where $N_{i, a}$ is the number of sites of stress level $s_{a}$ occupied by species $i, f_{i}$ is species $i$ 's per capita rate of seed production, $m_{i}$ is the adult mortality rate of species $i, Q_{i}\left(s_{a}\right)$ is the probability that one of species $i$ 's seeds fall on a site of stress level $s_{a}$ and recruits there, and $N_{i}$ is the total number of sites species $i$ occupies, i.e.

$$
N_{i}=\sum_{a=1}^{M} N_{i, a}
$$

The per-seed probability of successful recruitment $Q_{i}\left(s_{a}\right)$ is the product of three independent probabilities. First, we need to calculate the probability $G\left(s_{a}\right)$ that a seed arrives at an empty site of stress level $s_{a}$. This is given by

$$
G\left(s_{a}\right)=\frac{c\left(s_{a}\right)-\sum_{i=1}^{S} N_{i, a}}{\sum_{b=1}^{M} c\left(s_{b}\right)},
$$

where $c\left(s_{a}\right)$ is the number of sites of stress level $s_{a}$, so the numerator expresses the number of sites of stress $s_{a}$ that are not yet occupied, and the denominator is simply the total number of sites altogether. Second, once a seed arrives at a site, it has to survive the local stress level. Let us denote the probability that species $i$ 's seed survives stress level $s$ by $T_{i}(s)$ and call it the tolerance function (Figure S1A). It is assumed to be a decreasing function of stress level, and it is also assumed that species with higher fecundities $f_{i}$ are less tolerant of stress, which is the essence of the tolerance-fecundity tradeoff (Muller-Landau 2010, D'Andrea et al. 2013). Third, from the pool of seeds that arrived at a site of stress level $s_{a}$ and survived, one is chosen via lottery draw to win the site. Let $F\left(s_{a}\right)$ be the total number of seeds that survive on a site of stress level $s_{a}$. Then $1 / F\left(s_{a}\right)$ is the probability of winning the lottery draw. But $F\left(s_{a}\right)$ is simply given by

$$
F\left(s_{a}\right)=\frac{\sum_{k=1}^{S} f_{k} N_{k} T_{k}\left(s_{a}\right)}{\sum_{b=1}^{M} c\left(s_{b}\right)},
$$


the total number of surviving seeds divided by the total number of sites. Therefore, $Q_{i}\left(s_{a}\right)$ may be written

$$
Q_{i}\left(s_{a}\right)=G\left(s_{a}\right) T_{i}\left(s_{a}\right) \frac{1}{F\left(s_{a}\right)}=T_{i}\left(s_{a}\right) \frac{c\left(s_{a}\right)-\sum_{i=1}^{S} N_{i, a}}{\sum_{k=1}^{S} f_{k} N_{k} T_{k}\left(s_{a}\right)} .
$$

Substituting this expression into Eq. (S16) yields

$$
\frac{\mathrm{d} N_{i, a}}{\mathrm{~d} t}=f_{i} N_{i} T_{i}\left(s_{a}\right) \frac{c\left(s_{a}\right)-\sum_{i=1}^{S} N_{i, a}}{\sum_{k=1}^{S} f_{k} N_{k} T_{k}\left(s_{a}\right)}-m_{i} N_{i, a} .
$$

Let us cast this model equation in the form of traditional structured population models:

$$
\frac{\mathrm{d} N_{i, a}}{\mathrm{~d} t}=\sum_{b=1}^{M}\left(f_{i} T_{i}\left(s_{a}\right) \frac{c\left(s_{a}\right)-\sum_{i=1}^{S} N_{i, a}}{\sum_{k=1}^{S} f_{k} N_{k} T_{k}\left(s_{a}\right)}-m_{i} \delta_{a b}\right) N_{i, b}
$$

where we used Eq. (S17) in the first term on the right hand side, and the $\delta_{a b}$ in the second term is the identity matrix, equal to 1 if $a=b$ and to 0 otherwise. The expression in parentheses is the projection matrix $A_{i, a b}$ of species $i$, multiplying the stage distribution vector $N_{i, b}$ :

$$
A_{i, a b}=f_{i} T_{i}\left(s_{a}\right) \frac{c\left(s_{a}\right)-\sum_{i=1}^{S} N_{i, a}}{\sum_{k=1}^{S} f_{k} N_{k} T_{k}\left(s_{a}\right)}-m_{i} \delta_{a b}
$$

The model therefore describes a community of interacting structured populations in continuous time, where the $a$ th stage class of species $i$ measures the number of sites of stress level $s_{a}$ that species $i$ occupies.

\section{Sensitivity analysis}

For the tolerance-fecundity tradeoff model we are only performing qualitative sensitivity analysis, i.e., we calculate the impact and sensitivity vectors and the volumes they span but do not evaluate the full sensitivity formula.

Step 0. As demonstrated elsewhere numerically (D'Andrea et al. 2013), this model converges to a stable equilibrium state. We therefore wish to analyze the sensitivity of this fixed point, generated by the model which is in continuous time and involves population structure. The appropriate sensitivity formulas are therefore given by

$$
\sigma_{i}=\frac{\mathrm{d} N_{i}}{\mathrm{~d} \mathbb{E}}, \quad a_{i j}=\sum_{\mu} \underbrace{\left(\sum_{a, b} v_{i, a} \frac{\partial A_{i, a b}}{\partial \mathcal{R}_{\mu}} w_{i, b}\right)}_{\mathcal{S}_{i, \mu}} \underbrace{\left(\sum_{c} \frac{\partial \mathcal{R}_{\mu}}{\partial N_{j, c}} w_{j, c}\right)}_{\mathcal{I}_{j, \mu}}, \quad z_{j}=\sum_{a, b} v_{j, a} \frac{\partial A_{j, a b}}{\partial \mathbb{E}} w_{j, b}
$$

(Szilágyi \& Meszéna 2009, Barabás et al. 2014), where $v_{i, a}$ and $w_{i, a}$ are the $a$ th component of the left and right leading eigenvectors of $A_{i, a b}$, respectively. Note that these are simplified expressions; the 
fully general form (which we do not need here) is found in Eq. (35) of the main text or in Barabás et al. (2014). For these formulas to hold, the normalization conditions

$$
\sum_{a=1}^{M} w_{i, a}=1, \quad \sum_{a=1}^{M} v_{i, a} w_{i, a}=1
$$

have to be observed for all $i$. Biologically, since Eq. (S24) is evaluated at equilibrium, the left leading eigenvector is the reproductive value vector while the right leading eigenvector is the stable stage distribution.

In general we would need to solve the model for the equilibrium state. This model is complicated enough that this would only be possible using numerical simulations. However, as we will see, explicit knowledge of the equilibrium state is not needed to draw useful qualitative conclusions about the behavior of this system.

Step 1. We choose the regulating factors. Notice that the $A_{i, a b}$ depend on the densities only through the fraction in the first term of Eq. (S23). Therefore a natural choice for the regulating factors is

$$
\mathcal{R}\left(s_{a}\right)=\frac{c\left(s_{a}\right)-\sum_{i=1}^{S} N_{i, a}}{\sum_{k=1}^{S} f_{k} N_{k} T_{k}\left(s_{a}\right)}=\frac{G\left(s_{a}\right)}{F\left(s_{a}\right)},
$$

which is the density-dependent factor in $Q_{i}\left(s_{a}\right)$. The projection matrices then read

$$
A_{i, a b}=f_{i} T_{i}\left(s_{a}\right) \mathcal{R}\left(s_{a}\right)-m_{i} \delta_{a b},
$$

which is density-independent if we keep $\mathcal{R}\left(s_{a}\right)$ artificially fixed for all $a$, i.e., this is indeed a proper choice for the regulating factors.

In this model, space is the only limiting resource. However, space is not homogeneous, as sites differ in stress level. One therefore has to measure crowding for each stress level separately, resulting in $M$ different regulating factors, the $\mathcal{R}\left(s_{a}\right)$ for every $a$. If the gradation of various stress levels is infinitely fine-grained (i.e., we have a smooth stress gradient), $M \rightarrow \infty$ and we have infinitely many regulating factors, in perfect analogy with a resource continuum.

Step $2 a$. We first calculate the sensitivity vectors, and then the impact vectors (Step 2b). From Eq. (S24), the sensitivity of the $i$ th species to the $\mu$ th regulating factor reads

$$
\mathcal{S}_{i, \mu}=\sum_{a=1}^{M} \sum_{b=1}^{M} v_{i, a} \frac{\partial A_{i, a b}}{\partial \mathcal{R}\left(s_{\mu}\right)} w_{i, b}
$$

The derivative can be calculated directly using Eq. (S27):

$$
\frac{\partial A_{i, a b}}{\partial \mathcal{R}\left(s_{\mu}\right)}=\frac{\partial}{\partial \mathcal{R}\left(s_{\mu}\right)}\left(f_{i} T_{i}\left(s_{a}\right) \mathcal{R}\left(s_{a}\right)-m_{i} \delta_{a b}\right)=f_{i} T_{i}\left(s_{a}\right) \delta_{a \mu}
$$

To evaluate the eigenvectors of $A_{i, a b}$, notice first that the second term on the right hand side of Eq. (S27) is proportional to the identity matrix and does not influence the eigenvectors. Therefore, 
only the first term (let us denote it by $U_{a b}$ ) needs to be considered for calculating eigenvectors. This first term can also be written $U_{a b}=g_{a} h_{b}$, where $g_{a}=f_{i} T_{i}\left(s_{a}\right) \mathcal{R}\left(s_{a}\right)$ and $h_{b}=1$ (each component of $h_{b}$ is equal to 1 ). Such a matrix has only one right and corresponding left eigenvector (given by $g_{a}$ and $h_{b}$ themselves) such that their corresponding eigenvalue is nonzero. Indeed, for any vector $x_{a}$,

$$
\sum_{b=1}^{M} U_{a b} x_{b}=\sum_{b=1}^{M} g_{a} h_{b} x_{b}=g_{a}\left(\sum_{b=1}^{M} h_{b} x_{b}\right)
$$

therefore $x_{a}=g_{a}$ is the only right eigenvector with a nonzero eigenvalue. Similarly, $h_{a}$ is the only left eigenvector with a nonzero eigenvalue, because for any vector $x_{a}$,

$$
\sum_{b=1}^{M} x_{b} U_{b a}=\sum_{b=1}^{M} x_{b} g_{b} h_{a}=h_{a}\left(\sum_{b=1}^{M} g_{b} x_{b}\right) .
$$

From this it is also clear that the nonzero eigenvalue itself is given by $\sum_{a=1}^{M} g_{a} h_{a}$. As both $g_{a}$ and $h_{a}$ are, in our case, vectors with positive components, this eigenvalue must be some positive number. This means that, since all the other eigenvalues are zero, this eigenvalue is the leading one. Therefore, its left and right eigenvectors must correspond to the reproductive value and the stable stage distribution, respectively.

The stable stage distribution is then proportional to $g_{a}=f_{i} T_{i}\left(s_{a}\right) \mathcal{R}\left(s_{a}\right)$ :

$$
w_{i, a}=q_{i} f_{i} T_{i}\left(s_{a}\right) \mathcal{R}\left(s_{a}\right),
$$

where

$$
q_{i}=\left(\sum_{a=1}^{M} f_{i} T_{i}\left(s_{a}\right) \mathcal{R}\left(s_{a}\right)\right)^{-1}
$$

ensures proper normalization. Similarly, since $h_{a}=1$, the properly normalized left eigenvector is

$$
v_{i, a}=1
$$

for all species. Using Eqs. (S29), (S32), and (S34), the sensitivity vectors can now be calculated from the definition Eq. (S28):

$$
\begin{aligned}
\mathcal{S}_{i, \mu} & =\sum_{a=1}^{M} \sum_{b=1}^{M} v_{i, a} \frac{\partial A_{i, a b}}{\partial \mathcal{R}\left(s_{\mu}\right)} w_{i, b}=\sum_{a=1}^{M} \sum_{b=1}^{M} f_{i} T_{i}\left(s_{a}\right) \delta_{a \mu} q_{i} f_{i} T_{i}\left(s_{b}\right) \mathcal{R}\left(s_{b}\right) \\
& =\sum_{a=1}^{M} f_{i} T_{i}\left(s_{a}\right) \delta_{a \mu} q_{i} \underbrace{\left(\sum_{b=1}^{M} f_{i} T_{i}\left(s_{b}\right) \mathcal{R}\left(s_{b}\right)\right)}_{1 / q_{i}}=\sum_{a=1}^{M} f_{i} T_{i}\left(s_{a}\right) \delta_{a \mu}=f_{i} T_{i}\left(s_{\mu}\right) .
\end{aligned}
$$

In words, the sensitivity vector of species $i$ is its tolerance function weighted by its fecundity. This can be evaluated without any knowledge of the system's dynamics or current state. 
Step $2 b$. The formula for the impact vector of the $j$ th species on the $\mu$ th regulating factor in structured community models is given in Eq. (S24) as

$$
\mathcal{I}_{j, \mu}=\sum_{a=1}^{M} \frac{\partial \mathcal{R}\left(s_{\mu}\right)}{\partial N_{j, a}} w_{j, a}
$$

Using Eq. (S32), this becomes

$$
\mathcal{I}_{j, \mu}=\sum_{a=1}^{M} \frac{\partial \mathcal{R}\left(s_{\mu}\right)}{\partial N_{j, a}} q_{j} f_{j} T_{j}\left(s_{a}\right) \mathcal{R}\left(s_{a}\right)
$$

We calculate the partial derivative:

$$
\begin{aligned}
\frac{\partial \mathcal{R}\left(s_{\mu}\right)}{\partial N_{j, a}} & =-\frac{\delta_{a \mu}}{\sum_{k=1}^{S} f_{k} N_{k} T_{k}\left(s_{\mu}\right)}-\frac{c\left(s_{\mu}\right)-\sum_{i=1}^{S} N_{i, \mu}}{\left(\sum_{k=1}^{S} f_{k} N_{k} T_{k}\left(s_{\mu}\right)\right)^{2}} \underbrace{\frac{\partial}{\partial N_{j, a}}\left(\sum_{k=1}^{S} f_{k}\left(\sum_{b=1}^{M} N_{k, b}\right) T_{k}\left(s_{\mu}\right)\right)}_{f_{j} T_{j}\left(s_{\mu}\right)} \\
& =-\frac{\delta_{a \mu}}{\sum_{k=1}^{S} f_{k} N_{k} T_{k}\left(s_{\mu}\right)}-\frac{c\left(s_{\mu}\right)-\sum_{i=1}^{S} N_{i, \mu}}{\left(\sum_{k=1}^{S} f_{k} N_{k} T_{k}\left(s_{\mu}\right)\right)^{2}} f_{j} T_{j}\left(s_{\mu}\right),
\end{aligned}
$$

or, using Eq. (S26) in the second term,

$$
\frac{\partial \mathcal{R}\left(s_{\mu}\right)}{\partial N_{j, a}}=-\left(\frac{\delta_{a \mu}}{\sum_{k=1}^{S} f_{k} N_{k} T_{k}\left(s_{\mu}\right)}+\frac{f_{j} T_{j}\left(s_{\mu}\right) \mathcal{R}\left(s_{\mu}\right)}{\sum_{k=1}^{S} f_{k} N_{k} T_{k}\left(s_{\mu}\right)}\right) .
$$

The impact vectors then read

$$
\mathcal{I}_{j, \mu}=-\sum_{a=1}^{M}\left(\frac{\delta_{a \mu}}{\sum_{k=1}^{S} f_{k} N_{k} T_{k}\left(s_{\mu}\right)}+\frac{f_{j} T_{j}\left(s_{\mu}\right) \mathcal{R}\left(s_{\mu}\right)}{\sum_{k=1}^{S} f_{k} N_{k} T_{k}\left(s_{\mu}\right)}\right) q_{j} f_{j} T_{j}\left(s_{a}\right) \mathcal{R}\left(s_{a}\right),
$$

which can be written as

$$
\mathcal{I}_{j, \mu}=-\frac{q_{j} f_{j} T_{j}\left(s_{\mu}\right) \mathcal{R}\left(s_{\mu}\right)}{\sum_{k=1}^{S} f_{k} N_{k} T_{k}\left(s_{\mu}\right)}-\frac{f_{j} T_{j}\left(s_{\mu}\right) \mathcal{R}\left(s_{\mu}\right)}{\sum_{k=1}^{S} f_{k} N_{k} T_{k}\left(s_{\mu}\right)} q_{j} \sum_{a=1}^{M} f_{j} T_{j}\left(s_{a}\right) \mathcal{R}\left(s_{a}\right) .
$$

Since the last sum is simply equal to $1 / q_{j}$ due to Eq. (S33), the impact vectors are given by

$$
\mathcal{I}_{j, \mu}=-\frac{\left(q_{j}+1\right) f_{j} T_{j}\left(s_{\mu}\right) \mathcal{R}\left(s_{\mu}\right)}{\sum_{k=1}^{S} f_{k} N_{k} T_{k}\left(s_{\mu}\right)} .
$$

Step $3 a$. Here we calculate the volume spanned by the sensitivity vectors. In doing so, it is cleanest to assume there is an arbitrarily fine gradation of stress levels, therefore the sensitivity vector $\mathcal{S}_{i, \mu}=f_{i} T_{i}\left(s_{\mu}\right)$ becomes a smooth sensitivity function $\mathcal{S}_{i}(\mu)=f_{i} T_{i}(\mu)$. The volume $\mathcal{V}_{\mathcal{S}}$ 

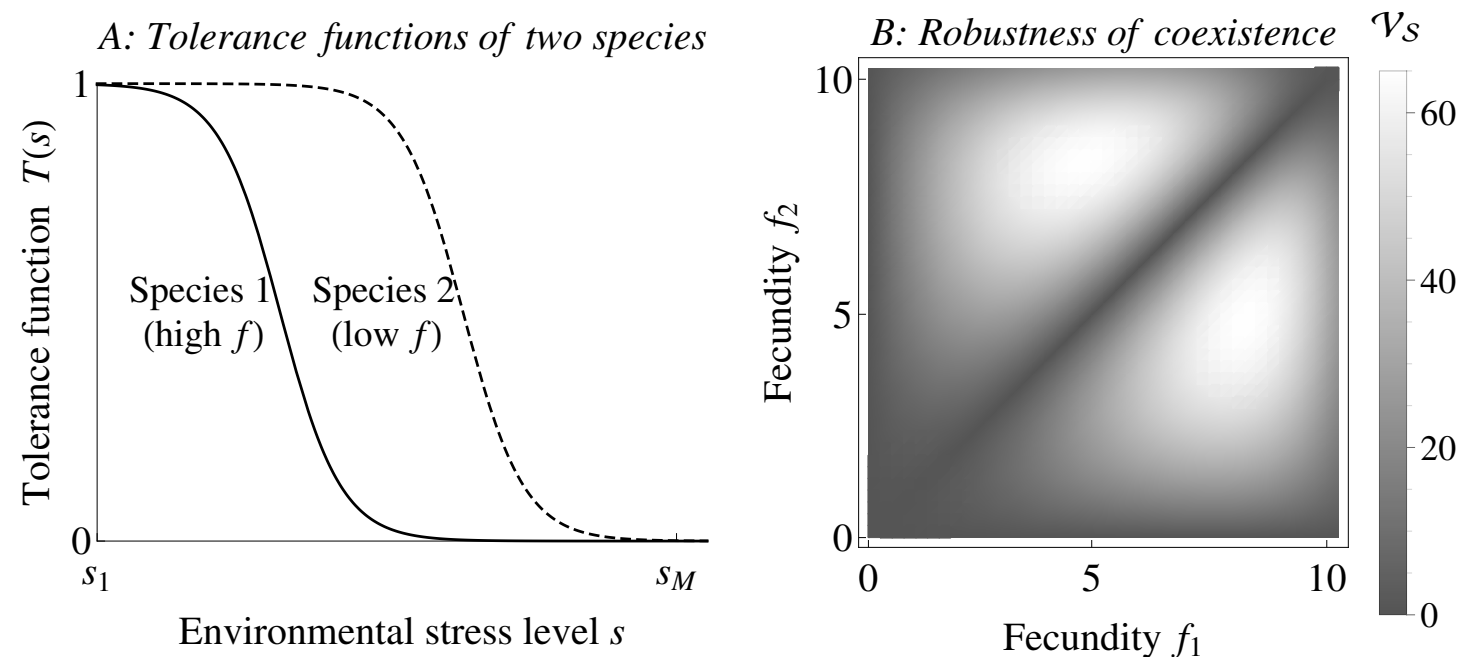

Fecundity $f_{1}$

Figure S1: Panel A shows the tolerance functions of two species (solid and dashed curves). The abscissa represents stress, ranging from $s_{1}$ (minimum level) to $s_{M}$ (maximum level). The ordinate is the probability that a seed survives the given stress level. The tolerance functions are sigmoid curves with a relatively abrupt transition from the tolerant to the intolerant regime. The tolerance-fecundity tradeoff is implemented by making the species with the higher fecundity $f$ less tolerant. Panel B shows the volume $\mathcal{V}_{\mathcal{S}}$ spanned by the sensitivity vectors of two species, as a function of their fecundities. The volume is largest where one species has a high fecundity and the other an intermediate one. Both species possessing similar fecundities leads to small volumes. Since a small volume is sufficient for making coexistence oversensitive and therefore unrealistic, it is only in the high-volume regions where coexistence is even a possibility.

(which is an area in our case because we consider $S=2$ ) spanned by two sensitivity functions $\mathcal{S}_{1}(\mu)$ and $\mathcal{S}_{2}(\mu)$ is given by Eq. (S69) as the determinant of the matrix of scalar products, taking its absolute value and square root (see the Appendix):

$$
\mathcal{V}_{\mathcal{S}}=\sqrt{\left|\left(\int_{s_{1}}^{s_{M}} \mathcal{S}_{1}^{2}(\mu) \mathrm{d} \mu\right)\left(\int_{s_{1}}^{s_{M}} \mathcal{S}_{2}^{2}(\mu) \mathrm{d} \mu\right)-\left(\int_{s_{1}}^{s_{M}} \mathcal{S}_{1}(\mu) \mathcal{S}_{2}(\mu) \mathrm{d} \mu\right)^{2}\right|}
$$

The particular form we use for the tolerance function is

$$
T_{i}(s)=\frac{\tanh \left(\tau\left(s_{M}-f_{i}-s\right)\right)+1}{2}
$$

shown on Figure S1A. 
We can evaluate the integrals. The indefinite integral of $\mathcal{S}_{i}^{2}(\mu)$ is

$$
\begin{aligned}
& \int \mathcal{S}_{i}^{2}(\mu) \mathrm{d} \mu=f_{i}^{2} \int T_{i}^{2}(\mu) \mathrm{d} \mu=\frac{f_{i}^{2}}{4} \int\left(\tanh \left(\tau\left(s_{M}-f_{i}-\mu\right)\right)+1\right)^{2} \mathrm{~d} \mu \\
& =-\frac{f_{i}^{2}}{4 \tau}\left(\tanh \left(\tau\left(f_{i}+\mu-s_{M}\right)\right)+2 \log \left(\cosh \left(\tau\left(f_{i}+\mu-s_{M}\right)\right)\right)-2 a \mu\right)+\text { const }
\end{aligned}
$$

as is easily verified by taking the expression's derivative with respect to $\mu$. The definite integral then reads

$$
\begin{aligned}
& \int_{s_{1}}^{s_{M}} \mathcal{S}_{i}^{2}(\mu) \mathrm{d} \mu=\frac{f_{i}^{2}}{4 \tau}\left(-\tanh \left(\tau\left(f_{i}\right)\right)-2 \log \left(\cosh \left(\tau\left(f_{i}\right)\right)\right)\right. \\
& \left.\quad+\tanh \left(\tau\left(f_{i}+s_{1}-s_{M}\right)\right)+2 \log \left(\cosh \left(\tau\left(f_{i}+s_{1}-s_{M}\right)\right)\right)+2 \tau_{s_{M}}-2 s_{1} \tau\right) .
\end{aligned}
$$

The other integral is

$$
\begin{aligned}
& \int \mathcal{S}_{1}(\mu) \mathcal{S}_{2}(\mu) \mathrm{d} \mu=\frac{f_{1} f_{2}}{4 \tau}\left(-\log \left(\cosh \left(\tau\left(f_{1}+\mu-s_{M}\right)\right)\right)-\log \left(\cosh \left(\tau\left(f_{2}+\mu-s_{M}\right)\right)\right)\right. \\
& \left.+\operatorname{coth}\left(\left(f_{1}-f_{2}\right) \tau\right)\left(\log \left(\cosh \left(\tau\left(f_{2}+\mu-s_{M}\right)\right)\right)-\log \left(\cosh \left(\tau\left(f_{1}+\mu-s_{M}\right)\right)\right)\right)+2 \mu \tau\right) \\
& + \text { const. }
\end{aligned}
$$

Its definite integral is then

$$
\begin{aligned}
& \int_{s_{1}}^{s_{M}} \mathcal{S}_{1}(\mu) \mathcal{S}_{2}(\mu) \mathrm{d} \mu=\frac{f_{1} f_{2}}{4 \tau}\left(-\log \left(\cosh \left(\tau\left(f_{1}+s_{M}-s_{M}\right)\right)\right)-\log \left(\cosh \left(\tau\left(f_{2}\right)\right)\right)\right. \\
& \quad-\operatorname{coth}\left(\left(f_{1}-f_{2}\right) \tau\right) \log \left(\cosh \left(\tau\left(f_{1}\right)\right)\right)+\operatorname{coth}\left(\left(f_{1}-f_{2}\right) \tau\right) \log \left(\cosh \left(\tau\left(f_{2}\right)\right)\right) \\
& \quad+\log \left(\cosh \left(\tau\left(f_{1}+s_{1}-s_{M}\right)\right)\right)+\log \left(\cosh \left(\tau\left(f_{2}+s_{1}-s_{M}\right)\right)\right) \\
& \quad+\operatorname{coth}\left(\left(f_{1}-f_{2}\right) \tau\right) \log \left(\cosh \left(\tau\left(f_{1}+s_{1}-s_{M}\right)\right)\right) \\
& \quad-\operatorname{coth}\left(\left(f_{1}-f_{2}\right) \tau\right) \log \left(\cosh \left(\tau\left(f_{2}+s_{1}-s_{M}\right)\right)\right) \\
& \left.\quad+2 \tau_{s_{M}}-2 s_{1} \tau\right) .
\end{aligned}
$$

Substituting Eqs. (S46) and (S48) into Eq. (S43), we get an explicit expression for $\mathcal{V}_{\mathcal{S}}$ as a function of the two fecundities $f_{1}$ and $f_{2}$ :

$$
\begin{aligned}
\mathcal{V}_{\mathcal{S}} & =\frac{f_{1} f_{2}}{4 \tau} \mid\left(-\tanh \left(\tau\left(f_{1}+s_{1}-s_{M}\right)\right)+2\left(-\log \left(\cosh \left(\tau\left(f_{1}+s_{1}-s_{M}\right)\right)\right)\right.\right. \\
& \left.\left.+\log \left(\cosh \left(f_{1} \tau\right)\right)+s_{1} \tau-s_{M} \tau\right)+\tanh \left(f_{1} \tau\right)\right)\left(-\tanh \left(\tau\left(f_{2}+s_{1}-s_{M}\right)\right)\right. \\
& \left.+2\left(-\log \left(\cosh \left(\tau\left(f_{2}+s_{1}-s_{M}\right)\right)\right)+\log \left(\cosh \left(f_{2} \tau\right)\right)+s_{1} \tau-s_{M} \tau\right)+\tanh \left(f_{2} \tau\right)\right) \\
& -\left(-\log \left(\cosh \left(\tau\left(f_{1}+s_{1}-s_{M}\right)\right)\right)-\log \left(\cosh \left(\tau\left(f_{2}+s_{1}-s_{M}\right)\right)\right)\right. \\
& +\operatorname{coth}\left(\left(f_{1}-f_{2}\right) \tau\right)\left(-\log \left(\cosh \left(\tau\left(f_{1}+s_{1}-s_{M}\right)\right)+\log \left(\cosh \left(\tau\left(f_{2}+s_{1}-s_{M}\right)\right)\right)\right.\right. \\
& \left.+\log \left(\cosh \left(f_{1} \tau\right)\right)-\log \left(\cosh \left(f_{2} \tau\right)\right)\right)+\log \left(\cosh \left(f_{1} \tau\right)\right)+\log \left(\cosh \left(f_{2} \tau\right)\right) \\
& \left.+2 s_{1} \tau-2 s_{M} \tau\right)\left.^{2}\right|^{1 / 2} .
\end{aligned}
$$


This is the function plotted on Figure S1B, with $s_{1}=0, s_{M}=10$, and $\tau=1$.

Step $3 b$. The volume spanned by the impact vectors. As Eq. (S42) depends on the stationary densities $N_{k}$, the volume $\mathcal{V}_{I}$ cannot be evaluated without knowing these quantities. However, this volume is not needed for drawing qualitative conclusions based on the sensitivity vectors only, for the following reason.

In Eq. (S42), $T_{j}\left(s_{\mu}\right)$ and $\mathcal{R}\left(s_{\mu}\right)$ in the numerator are are quantities whose values fall between 0 and 1 . The denominator expresses the seed rain, and since we have made the assumption at the outset that the seed rain contains at least one seed per empty site, the denominator cannot be smaller than 1 . The magnitude of any one component of the $j$ th sensitivity vector therefore cannot exceed $\left(q_{j}+1\right) f_{j}$. There is no reason why this factor should be very large: $f_{j}$ is a rate and so proportional to the log of the annual seed production, while $q_{j}$ is simply a normalizing constant. This means that every component of each impact vector is bounded in magnitude. Therefore, the volume spanned by these vectors will also be bounded.

Since robustness is determined by $\mathcal{V}_{\mathcal{I}} \mathcal{V}_{\mathcal{S}}$, this observation means that whenever the volume spanned by the sensitivity vectors is small, robustness will also necessarily be small, because $\mathcal{V}_{\mathcal{I}}$ is bounded from above and so cannot compensate for a small $\mathcal{V}_{\mathcal{S}}$.

Moreover, we can see from Eq. (S42) that $\mathcal{I}_{j, \mu}$ is actually proportional to $\mathcal{S}_{j, \mu}=f_{j} T_{j}\left(s_{\mu}\right)$, therefore $\mathcal{V}_{\mathcal{I}}$ will be large/small wherever $\mathcal{V}_{\mathcal{S}}$ is large/small.

This means that the volumes spanned by the sensitivity vectors are sufficient to determine the robustness of coexistence in the sense that wherever the sensitivity volume is small, coexistence cannot be expected to hold. 


\section{The Gross model}

\section{Model description}

In the model of Gross (2008), there is a single resource and several consumer species. The consumers have facilitative effects on one another: an increase in the abundance of one species reduces the death rate of another. The general form of these death rates is chosen to be

$$
m_{i}=m_{i}^{0}-d_{i}\left(1-\exp \left(-\sum_{k=1}^{S} \theta_{i k} N_{k}\right)\right),
$$

where $S$ is the total number of consumer species, $m_{i}^{0}$ is the baseline mortality of species $i, d_{i}$ is the maximum advantage it can gain from facilitation (we assume $d_{i} \leq m_{i}^{0}$ ), $N_{k}$ is the density of species $k$, and $\theta_{i k}$ is a matrix of scaling factors, measuring the benefit species $k$ confers to species $i$. Since there is no self-facilitation, the diagonal elements are all zero: $\theta_{i i}=0$ for all $i=1 \ldots S$. Using these mortalities, the model equations are written

$$
r_{i}=\frac{1}{N_{i}} \frac{\mathrm{d} N_{i}}{\mathrm{~d} t}=f_{i}(R)-m_{i} \quad(i=1 \ldots S)
$$

for the species ( $r_{i}$ is the per capita growth rate of species $i$ as always), and

$$
\frac{\mathrm{d} R}{\mathrm{~d} t}=g(R)-\sum_{i=1}^{S} c_{i} f_{i}(R) N_{i}
$$

for the resource. Here $f_{i}(R)$ is the per capita resource-dependent growth rate of species $i$, the $m_{i}$ are given by Eq. (S50), $R$ is the amount of resource in the system, $g(R)$ is the resource supply rate, and the $c_{i}$ measure the amount of resource species $i$ has to consume to produce one unit of biomass.

The form of the mortalities in Eq. (S50) is fairly general. Gross (2008) made the assumption of hierarchical facilitation to narrow it further down. This is implemented by choosing $\theta_{i k}$ to be zero for $k \geq i$ and a positive constant otherwise:

$$
m_{i}=m_{i}^{0}-d_{i}\left(1-\exp \left(-\theta \sum_{k<i} N_{k}\right)\right) .
$$

Notice that, since the sum only runs through species $k<i$, species $i$ is facilitated only by those who have a lower species index: species 1 is not facilitated by anyone, species 2 is facilitated by species 1 , species 3 is facilitated by species 1 and 2 , and so on. Also, the advantage a species receives (if any) from a single other species is always the same, $\theta$.

\section{Sensitivity analysis}

Here we perform qualitative sensitivity analysis again, calculating the product of the volumes spanned by the impact and sensitivity vectors, and showing that this product converges to zero for large $S$. 
Step 0. It was already proved by Gross (2008) that this system has a stable all-positive fixed point. In the two-species case, limit cycles were also observed. While they cannot be ruled out for $S>2$, they have not been observed in numerical simulations. Even if there happen to be alternative attractors in phase space, we will concentrate on the sensitivity of the nontrivial fixed point only. Fortunately, as we will see, an explicit knowledge of this equilibrium state will not be needed for our analysis.

Since the model is unstructured, continuous time, and we are analyzing the sensitivity of a fixed point, the appropriate sensitivity formulas are given by

$$
\sigma_{i}=\frac{\mathrm{d} N_{i}}{\mathrm{~d} \mathbb{E}}, \quad a_{i j}=\sum_{\mu} \underbrace{\frac{\partial r_{i}}{\partial \mathcal{R}_{\mu}}}_{\mathcal{S}_{i, \mu}} \underbrace{\frac{\partial \mathcal{R}_{\mu}}{\partial N_{j}}}_{\mathcal{I}_{j, \mu}}, \quad z_{j}=\frac{\partial r_{j}}{\partial \mathbb{E}}
$$

(Meszéna et al. 2006).

Step 1 . We need to designate the regulating factors. We make the following choice:

$$
\begin{aligned}
\mathcal{R}_{1} & =R \\
\mathcal{R}_{\mu} & =\exp \left(-\theta \sum_{k<\mu} N_{k}\right) \quad(\mu=2 \ldots S) .
\end{aligned}
$$

Note that we could have made other choices as well-we could have made $\mathcal{R}_{\mu>1}=\sum_{k<\mu} N_{k}$ or even just $\mathcal{R}_{\mu>1}=N_{\mu}$, as all these choices satisfy the criterion that keeping their values artificially fixed leads to the density-independence of the growth rates $r_{i}$. However, performing the necessary calculations may be easy with some choices and hard or even impossible with others. In our case, it turns out that Eq. (S55) lends itself to analytical treatment much better than the other choices (see below). The model equations, rewritten in terms of the regulating factors, are

$$
\begin{aligned}
r_{1} & =f_{1}\left(\mathcal{R}_{1}\right)-m_{1}^{0}, \\
r_{i} & =f_{i}\left(\mathcal{R}_{1}\right)-m_{i}^{0}+d_{i}\left(1-\mathcal{R}_{i}\right) \quad(i=2 \ldots S) .
\end{aligned}
$$

We can see that our choice of regulating factors is valid, because fixing their values would make the per capita growth rates density-independent.

Step 2. The impact vectors $\mathcal{I}_{j, \mu}$ and sensitivity vectors $\mathcal{S}_{i, \mu}$ are calculated using Eq. (S54):

$$
\begin{aligned}
\mathcal{I}_{j, \mu}=\frac{\partial \mathcal{R}_{\mu}}{\partial N_{j}}=(\frac{\partial \mathcal{R}_{1}}{\partial N_{j}}, 0, \ldots, 0, \underbrace{-\theta \mathcal{R}_{j+1}}_{\mu=j+1},-\theta \mathcal{R}_{j+2}, \ldots,-\theta \mathcal{R}_{S}), \\
\mathcal{S}_{i, \mu}=\frac{\partial r_{i}}{\partial \mathcal{R}_{\mu}}=(\frac{\partial f_{i}}{\partial \mathcal{R}_{1}}, 0, \ldots, 0, \underbrace{-d_{i}}_{\mu=i}, 0, \ldots, 0),
\end{aligned}
$$

where all quantities are evaluated at equilibrium. 
Step 3. We calculate the two volumes. This is where our particular choice for the regulating factors proves useful: it would be impossible to do the calculation using other choices. The volumes are calculated via Eq. (S70) (see the Appendix). The impact volume reads

$$
\mathcal{V}_{\mathcal{I}}=\operatorname{det}\left(\begin{array}{cccc}
\frac{\partial \mathcal{R}_{1}}{\partial N_{1}} & -\theta \mathcal{R}_{2} & -\theta \mathcal{R}_{3} & \cdots \\
\frac{\partial \mathcal{R}_{1}}{\partial N_{2}} & 0 & -\theta \mathcal{R}_{3} & \ldots \\
\frac{\partial \mathcal{R}_{1}}{\partial N_{3}} & 0 & 0 & \ldots \\
\vdots & \vdots & \vdots & \ddots
\end{array}\right)|=| \frac{\partial \mathcal{R}_{1}}{\partial N_{S}} \mid \theta^{S-1} \prod_{i=2}^{S} \mathcal{R}_{i},
$$

and the sensitivity volume is

$$
\mathcal{V}_{\mathcal{S}}=\left|\operatorname{det}\left(\begin{array}{cccc}
\frac{\partial f_{1}}{\partial \mathcal{R}_{1}} & 0 & 0 & \cdots \\
\frac{\partial f_{2}}{\partial \mathcal{R}_{1}} & -d_{2} & 0 & \ldots \\
\frac{\partial f_{3}}{\partial \mathcal{R}_{1}} & 0 & -d_{3} & \ldots \\
\vdots & \vdots & \vdots & \ddots
\end{array}\right)\right|=\left|\frac{\partial f_{1}}{\partial \mathcal{R}_{1}}\right| \prod_{i=2}^{S} d_{i}
$$

The especially simple form of these matrices allowed for the direct calculation of the determinants.

The product of these volumes is

$$
\begin{aligned}
\mathcal{V}_{I} \mathcal{V}_{\mathcal{S}} & =\left|\frac{\partial f_{1}}{\partial \mathcal{R}_{1}} \frac{\partial \mathcal{R}_{1}}{\partial N_{S}}\right| \theta^{S-1}\left(\prod_{i=2}^{S} d_{i}\right)\left(\prod_{i=2}^{S} \mathcal{R}_{i}\right) \\
& =\left|\frac{\partial f_{1}}{\partial \mathcal{R}_{1}} \frac{\partial \mathcal{R}_{1}}{\partial N_{S}}\right| \theta^{S-1}\left(\prod_{i=2}^{S} d_{i}\right) \mathrm{e}^{-\theta N_{1}} \mathrm{e}^{-\theta\left(N_{1}+N_{2}\right)} \cdots \mathrm{e}^{-\theta\left(N_{1}+\cdots+N_{S-1}\right)} \\
& =\left|\frac{\partial f_{1}}{\partial \mathcal{R}_{1}} \frac{\partial \mathcal{R}_{1}}{\partial N_{S}}\right| \theta^{S-1}\left(\prod_{i=2}^{S} d_{i}\right) \mathrm{e}^{-\theta\left((S-1) N_{1}+(S-2) N_{2}+\cdots+N_{S-1}\right)}
\end{aligned}
$$

Let $N$ be the smallest of the equilibrium densities $N_{1}, N_{2}, \ldots, N_{S-1}$. Then we can write the inequality

$$
\begin{aligned}
\mathcal{V}_{\mathcal{I}} \mathcal{V}_{\mathcal{S}} & \leq\left|\frac{\partial f_{1}}{\partial \mathcal{R}_{1}} \frac{\partial \mathcal{R}_{1}}{\partial N_{S}}\right| \theta^{S-1}\left(\prod_{i=2}^{S} d_{i}\right) \mathrm{e}^{-\theta((S-1) N+(S-2) N+\cdots+N)} \\
& =\left|\frac{\partial f_{1}}{\partial \mathcal{R}_{1}} \frac{\partial \mathcal{R}_{1}}{\partial N_{S}}\right| \theta^{S-1}\left(\prod_{i=2}^{S} d_{i}\right) \mathrm{e}^{-N \theta S(S-1) / 2} .
\end{aligned}
$$

This expression asymptotically depends on the number of species as $\exp \left(-N \theta S^{2} / 2\right)$. It converges to zero faster than exponential in the number of species (unless $N$ decreases even faster-but in 
that case the equilibrium population densities would soon get so close to zero that, from a practical point of view, extinctions would be inevitable). Then, due to the above inequality, the product of the volumes spanned by the impact and sensitivity vectors must also converge to zero at least as fast as $\exp \left(-N \theta S^{2} / 2\right)$.

Note also that in this model, the product of the volumes is exactly equal to the determinant of the generalized community matrix $a_{i j}$ in absolute value. From Eq. (S54), this determinant can be written

$$
\operatorname{det}\left(a_{i j}\right)=\operatorname{det}\left(\sum_{\mu=1}^{S} \frac{\partial r_{i}}{\partial \mathcal{R}_{\mu}} \frac{\partial \mathcal{R}_{\mu}}{\partial N_{j}}\right)=\operatorname{det}\left(\frac{\partial r_{i}}{\partial \mathcal{R}_{\mu}}\right) \operatorname{det}\left(\frac{\partial \mathcal{R}_{\mu}}{\partial N_{j}}\right)=\operatorname{det}\left(\mathcal{S}_{i, \mu}\right) \operatorname{det}\left(\mathcal{I}_{j, \mu}\right),
$$

where we used two facts: 1) that the number of regulating factors happens to be equal to the number

of species and therefore $\mathcal{I}_{j, \mu}$ and $\mathcal{S}_{i, \mu}$ are square matrices, and 2) that the determinant of a product of square matrices is the product of the determinants. Due to the well-known geometrical interpretation of the determinant (see also the Appendix),

$$
\left|\operatorname{det}\left(a_{i j}\right)\right|=\left|\operatorname{det}\left(\mathcal{I}_{j, \mu}\right) \operatorname{det}\left(\mathcal{S}_{i, \mu}\right)\right|=\mathcal{V}_{\mathcal{I}} \mathcal{V}_{\mathcal{S}},
$$

which is what we wanted to show.

\section{The bound on the number of coexisting species}

We have shown above that the product $\mathcal{V}_{\mathcal{I}} \mathcal{V}_{\mathcal{S}}$-and therefore, due to Eq. (S64), the determinant of $a_{i j}$ - converges to zero as $S$ increases. It is tempting to jump to the conclusion that coexistence of a large number of species is extremely unlikely. Some additional care is needed, however: since introducing a new species will also increase the dimensionality of the problem, $\mathcal{V}_{\mathcal{I}} \mathcal{V}_{\mathcal{S}}$ approaching zero does not automatically imply decreasing robustness.

To illustrate why, consider $S$ noninteracting species. Assume each of them may have a mortality rate between 1 and 2, and only species with mortality lower than 1.5 are viable. The parameter range allowing for the persistence of a single species is 0.5 . For $S$ independent species, the total parameter volume allowing for the persistence of all species is then equal to $0.5^{S}$, which converges to zero for large $S$. Yet, the robustness of the community need not be vanishing: if, for instance, all species have a mortality of 1.25 , then no species will go extinct in response to small perturbations of any of these mortalities. Indeed, it is intuitively obvious that the number 0.5 is the "true" measure of robustness here, which is the $S$ th root of the total volume $0.5^{S}$.

To avoid measuring the trivial artifact of parameter volumes shrinking due to an increasing number of species, we observe from Eq. (S62) that the Sth root of the determinant still converges to zero exponentially:

$$
\sqrt[S]{\mathcal{V}_{\mathcal{I}} \mathcal{V}_{\mathcal{S}}} \sim \sqrt[S]{\theta^{S} \exp \left(-N \theta S^{2} / 2\right)}=\theta \exp (-N \theta S / 2) \rightarrow 0
$$

(only the leading term in $S$ was kept in the exponents). That is, the robustness of the community is still lost for large $S$, even when accounting for the above trivial effect. 
The above intuitive argument can be pitched more formally. The determinant of a matrix is the product of its eigenvalues. The eigenvalues describe the robustness of the community in specific directions, while their product measures overall robustness. An eigenvalue that is nearly zero means that the corresponding eigendirection is extremely sensitive, since a small perturbation could push that eigenvalue over to the right half plane, destabilizing the system. If the product of the eigenvalues approaches zero, this need not mean that any one particular eigenvalue does-e.g., if each eigenvalue is equal to 0.5 , none of them are very small, but their product does converge to zero for large $S$. But now let us consider not just the determinant, but the $S$ th root of the determinant. This quantity is simply the geometric mean of the eigenvalues. If the geometric mean goes to zero, then the smallest eigenvalue must also approach zero, as the geometric mean is necessarily greater than or equal to the smallest eigenvalue. ${ }^{\dagger}$ That is, when the Sth root of the determinant becomes small, one can be certain that robustness is lost in some of the individual directions as well. In our case, we can see from Eq. (S65) that this is exactly what happens.

From Eq. (S65) we can see that $S$ cannot be much larger than $1 /(N \theta)$, otherwise the likelihood of coexistence becomes exponentially small. To make this conclusion more transparent, let us consider a dimensional analysis of robustness. If $N$ is measured in units of biomass, then $\theta$ is of dimension inverse biomass. Their product $N \theta$ is then a dimensionless quantity. We may rescale Eq. (S65) by multiplying it with $N$ :

$$
\sqrt[S]{\mathcal{V}_{I} \mathcal{V}_{\mathcal{S}}} \sim N \theta \exp (-N \theta S / 2)
$$

This expression will still converge to zero whenever $S$ is large, and-unlike Eq. (S65)-is a dimensionless measure of robustness. It clearly converges to zero exactly when Eq. (S65) does; all we have done is a rescaling of the expression by the constant $N$. In this form it is clear that robustness is lost whenever the dimensionless quantity $N \theta$ is much larger or much smaller than 1 . Therefore, $S$ cannot be much larger than 1. (See also Meszéna et al. 2006, Eq. 52 for the calculation of the probability of coexistence as a dimensionless quantity, using the non-dimensionless determinant.)

Figure $\mathrm{S} 2$ plots the right hand side of Eq. (S66) for fixed values of $S$ as a function of $N \theta$. The curves reach their unique maximum at $N \theta=2 / S$; the value at the maximum is $2 /(\mathrm{e} S)$, as is easily seen by differentiating Eq. (S66) with respect to $N \theta$ and requiring the result to be zero. Therefore $\sqrt[S]{\mathcal{V}_{I} \mathcal{V}_{\mathcal{S}}}$ goes to zero with the inverse of $S$ even if one manages to fine-tune the parameters such that $N \theta$ remains at its optimal value for each $S$ - a fairly unrealistic situation to begin with.

Our take-home message is that the coexistence of more than a few species through the cascade of facilitation in the Gross model is a highly unlikely outcome. Moreover, this result was obtained by a simple argument which did not take variation in specific parameters into account. More specific evaluation of the coexistence bandwidth for any fixed value of the species number could be carried out like we did in the case of the Levine-Rees model.

\footnotetext{
$\dagger$ One might worry that the eigenvalues may be complex numbers. How do we interpret "smaller" or "greater" in this case? The problem is easily resolved: since $a_{i j}$ is necessarily a real matrix, its eigenvalues come in complex conjugate pairs, whose product is $(a+\mathrm{i} b)(a-\mathrm{i} b)=a^{2}+b^{2}=(\text { length })^{2}$. So in this case it is the length of the shortest eigenvalue that will approach zero.
} 


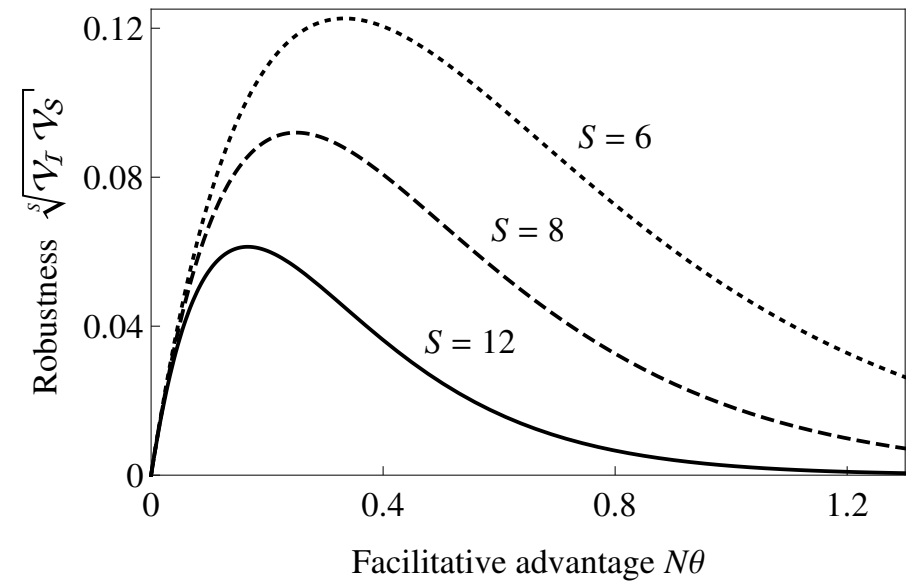

Figure S2: The robustness $\sqrt[S]{\mathcal{V}_{\mathcal{I}} \mathcal{V}_{\mathcal{S}}}$ as a function of the (scaled) facilitative advantage $N \theta$ for various values of $S$, based on Eq. (S66). The most robust scenario always happens at an intermediate $N \theta$ value.

\section{Appendix: the volume spanned by a set of vectors}

Suppose we are given a set of $S$ vectors, with $\mathcal{W}_{i, \mu}$ referring to the $\mu$ th entry of the $i$ th vector $(i=1 \ldots S)$. We assume without loss of generality that $\mu=1 \ldots L$, i.e., each vector has $L$ entries $(L$ is not necessarily equal to $S$ ). To calculate the volume $\mathcal{V}_{\mathcal{W}}$ of the parallelotope spanned by these vectors, define the following matrix of scalar products:

$$
W_{i j}=\sum_{\mu=1}^{L} W_{i, \mu} W_{j, \mu}
$$

Note that, in case $\mu$ is a continuous as opposed to a discrete index (i.e., when we have functions $\mathcal{W}_{i}(\mu)$ instead of vectors $\left.\mathcal{W}_{i, \mu}\right)$, the summation above becomes integration with respect to $\mu$ :

$$
W_{i j}=\int_{\mu_{0}}^{\mu_{1}} \mathcal{W}_{i}(\mu) \mathcal{W}_{j}(\mu) \mathrm{d} \mu
$$

where $\mu_{0}$ and $\mu_{1}$ are the appropriate limits of integration. Using $W_{i j}$, the volume $\mathcal{V}_{\mathcal{W}}$ may now be calculated with the help of the so-called Gram determinant $\operatorname{det}\left(W_{i j}\right)$ (Gantmacher 1959):

$$
\mathcal{V}_{\mathcal{W}}=\sqrt{\left|\operatorname{det}\left(W_{i j}\right)\right|}
$$

There are two special cases for which this expression can be simplified. First, $\mathcal{V}_{\mathcal{W}}=0$ whenever $L<S$. Second, for $L=S$, the formula simplifies to

$$
\mathcal{V}_{\mathcal{W}}=\left|\operatorname{det}\left(\mathcal{W}_{i, \mu}\right)\right|
$$

(Meszéna et al. 2006, Appendix C), where $\mathcal{W}_{i, \mu}$ is treated as a (square) matrix with row index $i$ and column index $\mu$. 


\section{References}

Barabás, G., Meszéna, G. \& Ostling, A. (2012). Community robustness and limiting similarity in periodic environments. Theoretical Ecology, 5, 265-282.

Barabás, G. \& Ostling, A. (2013). Community robustness in discrete-time periodic environments. Ecological Complexity, 15, 122-130.

Barabás, G., Meszéna, G. \& Ostling, A. (2014). Fixed point sensitivity analysis of interacting structured populations. Theoretical Population Biology, 92, 97-106.

Chesson, P. \& Warner, R. R. (1981). Environmental variability promotes coexistence in lottery competitive systems. American Naturalist, 117, 923-943.

Chesson, P. (1994). Multispecies competition in variable environments. Theoretical Population Biology, 45, 227-276.

Chesson, P. (2000). Mechanisms of maintenance of species diversity. Annual Review of Ecology and Systematics, 31, 343-366.

D’Andrea, R., Barabás, G. \& Ostling, A. (2013). Revising the tolerance-fecundity trade-off; or, on the consequences of discontinuous resource use for limiting similarity, species diversity, and trait dispersion. American Naturalist, 181, E91-101.

Gantmacher, F. R. (1959). Matrix theory. Chelsea Publishing, New York.

Gross, K. (2008). Positive interactions among competitors can produce species-rich communities. Ecology Letters, 11, 929-936.

Levine, J. M. \& Rees, M. (2004). Effects of temporal variability on rare plant persistence in annual systems. American Naturalist, 164, 350-363.

Meszéna, G., Gyllenberg, M., Pásztor, L. \& Metz, J. A. J. (2006). Competitive exclusion and limiting similarity: a unified theory. Theoretical Population Biology, 69, 68-87.

Muller-Landau, H. C. (2010). The tolerance-fecundity trade-off and the maintenance of diversity in seed size. Proceedings of the National Academy of Sciences of the USA, 107, 4242-4247.

Szilágyi, A. \& Meszéna, G. (2009). Limiting similarity and niche theory for structured populations. Journal of Theoretical Biology, 258, 27-37. 